\title{
INTEGRAL detection of a long powerful burst from SLX 1735-269^
}

\author{
S. Molkov ${ }^{1,2}$, M. Revnivtsev ${ }^{1,2}$, A. Lutovinov ${ }^{1}$, and R. Sunyaev ${ }^{1,2}$ \\ ${ }^{1}$ Space Research Institute, Russian Academy of Sciences, Profsoyuznaya 84/32, 117997 Moscow, Russia \\ e-mail: molkov@hea.iki.rssi.ru \\ 2 Max-Plank-Institute für Astrophysik, Karl-Schwarzschild-Str. 1, 85740 Garching bei München, Germany
}

Received 13 August 2004 / Accepted 21 December 2004

\begin{abstract}
We present results of an analysis of the bursting behavior of the low mass X-ray binary system SLX 1735-269 during INTEGRAL observations of the Galactic Center region in 2003. Six type-I X-ray bursts were detected in total with one being much longer and more powerful then the others. A strong dependence of the burst recurrence time on the mass accretion rate is observed, that is likely caused by a change in the burning regime. The long burst demonstrated a photospheric radius expansion. We discuss possible scenarios of this long burst and show that it is unlikely a carbon burning flash but rather burning of a large pile of hydrogen and helium accelerated by electron capture processes in a dense accumulated layer.
\end{abstract}

Key words. X-rays: binaries - X-rays: individuals: SLX 1735-269

\section{Introduction}

SLX 1735-269 was discovered as a persistent X-ray source in the energy range of $3-30 \mathrm{keV}$ in 1985 with the Spacelab 2 mission (Skinner et al. 1987). Since then the source was seen by different instruments with a flux of (2-5) $\times 10^{-10} \mathrm{erg} \mathrm{cm}^{-2} \mathrm{~s}^{-1}$ (Skinner et al. 1987; Pavlinsky et al. 1992, 1994; in 't Zand 1992; Grebenev et al. 1996). SLX $1735-269$ was detected in hard X-rays $(35-75 \mathrm{keV})$ by the SIGMA telescope on board the GRANAT observatory in 1992 (Goldwurm et al. 1996). A broadband spectral analysis of the system showed that it is likely a low mass X-ray binary (David et al. 1997). But only in 1997 was its nature finally established after a detection of a type I X-ray burst with the Wide Field Camera on the BeppoSAX observatory (Bazzano et al. 1997; Cocchi et al. 1998). The detection of this type I burst (unstable nuclear burning on the neutron star surface, see e.g. Hansen \& van Horn 1975) demonstrated that SLX 1735-269 is a neutron star binary system.

The burst detected with BeppoSAX/WFC remained the only one known from this system until the launch of the INTEGRAL satellite. The long exposure time spent by INTEGRAL on observations of the Galactic Center region allowed us to detect more bursts from SLX 1735-269. One of 6 detected bursts had a duration of more than $1000 \mathrm{~s}$ which is not typical for standard hydrogen/helium type I bursts but rather similar to so-called superbursts - unstable burning of

^ Based on observations with INTEGRAL, an ESA project with instruments and science data centre funded by ESA member states (especially the PI countries: Denmark, France, Germany, Italy, Switzerland, Spain), Czech Republic and Poland, and with the participation of Russia and the USA. carbon (see e.g. Cornelisse et al. 2000; Cumming \& Bildsten 2001; Strohmayer \& Brown 2002; in 't Zand et al. 2004).

In this paper we present the analysis of the bursting behavior of SLX 1735-269 concentrating on the properties of the unusually long burst.

\section{Instruments and observations}

In this work we present results from the JEM-X monitor, module 2 (see Lund et al. 2003) and the upper layer of the IBIS telescope (ISGRI/IBIS; Ubertini et al. 2003) of the INTEGRAL observatory (Winkler et al. 2003a). These instruments together cover a broad energy range of 3-400 keV with typical sensitivities of $\sim 4$ and $\sim 1.5 \mathrm{mCrab}$ for one orbit ( $\sim 3$ days) of observations with JEM-X (unit 2) and IBIS/ISGRI, respectively.

In 2003 the Galactic Center region was observed many times with INTEGRAL during both Open and Core Programs of the observatory (Winkler et al. 2003b). In our analysis we used the Galactic Center region observations of the Open Program performed in Aug. 23-Sept. 24, 2003 (proposal ID 0120213) and publicly available data of Galactic Center observations performed as part of the Core Program in Mar. 2Apr. 30, 2003. The total effective exposure of SLX1735-269 for the JEM-X telescope was $\sim 1.8 \mathrm{Ms}$, for the IBIS telescope 3.4 Ms. The smaller effective exposure for JEM-X is due to the dithering technique applied to most INTEGRAL observations which frequently moves the source outside the relatively small field of view of this instrument.

Type I bursts have a very soft $\mathrm{X}$-ray spectrum and the main part of its luminosity is emitted in the energy range 3-10 keV. Therefore, we used for the search of X-ray bursts the photon count rate over the whole JEM-X detector. 
JEM-X has five different telemetry formats: Full Imaging, Restricted Imaging, Spectral/Timing, Spectral and Timing. Usually it operates in the "Full Imaging mode", when the data are downloaded with a time resolution of $122 \mu \mathrm{s}$ and an energy resolution of 256 channels. This is the preferred mode, but JEM-X has a rather limited telemetry budget and sometimes is forced to switch to other modes. The photon count rate on its detector is dominated by cosmic sources and, therefore, the telemetry load depends strongly on the brightness of the sources in its field of view (FOV). If the count rate of the detector exceeds a limiting value then the on-board memory buffer reserved for JEM-X is overloaded and the information about all registered events cannot be transmitted to Earth. In order to avoid data gaps a grey filter is applied. Depending on the detector count rate this algorithm rejects " $n$ " from " 32 " events. Thus, the real count rate equals to the transmitted count rate multiplied by $32 /(1+G)$, where $G$ is a grey filter value, which can vary from " 0 " to " 31 ". If this algorithm cannot keep the count rate below the threshold, the restricted imaging mode is used. It has a time resolution of $0.125 \mathrm{~s}$ and 8 broad energy channels covering the whole JEM-X energy band. The grey filter algorithm can be applied to this mode also, which leads to a significant worsening of the time resolution.

During our observations JEM-X was mainly in the "full imaging mode" but a few times it switched to the "restricted imaging mode"

\section{Data reduction}

The analysis of the JEM-X telescope data was done using the standard INTEGRAL Off-line Science Analysis software version 4.0 (OSA-4.0) distributed by the INTEGRAL Science Data Center.

The JEM-X analysis package from OSA-4.0 has twelve steps of data treatment (see Chernyakova \& Kretschmar 2004, Part II). Below we discuss only three main levels: COR, IMA and SPE. COR is the first level of scientific analysis where events after a primary telemetry pre-processing are corrected for instrumental effects, such as energy gain correction and positional gain correction. An analysis up to IMA and SPE levels is based on the output data of the COR level. After the steps IMA and SPE we reconstructed images of the sky and spectra of detected sources. For the following study we combined outputs of all three levels listed above.

The OSA software is still under development and our investigations showed that a quantitative study of sources is not possible from the imaging. The measured absolute values of source fluxes are not fully reliable while their detection and location are. Therefore we used the JEM-X imaging analysis only for the burst localization.

For the timing analysis we used the data corrected for the instrumental effects (COR level, see above). Lightcurves were constructed from total count rate of the JEM-X detector in the required energy bands after correction for the grey filter factor. As the detector background only slightly varies with the time we used calibration observations of "empty fields" in order to determine the background level. To avoid problems with a vignetting correction in the following analysis we used only data

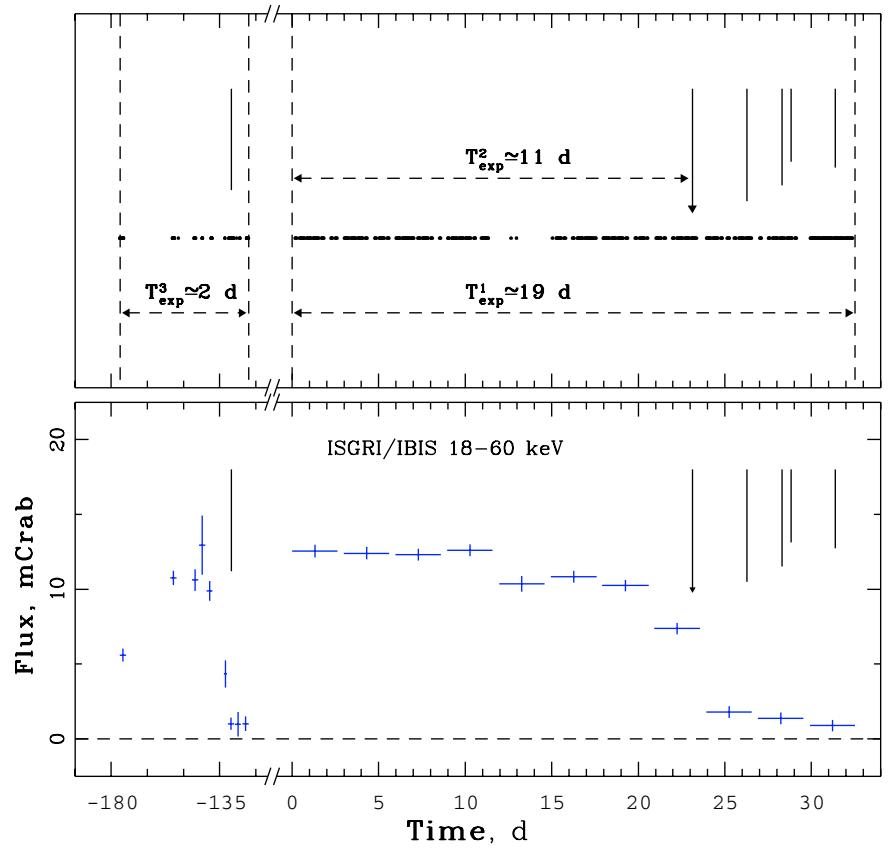

Fig. 1. History of INTEGRAL observations of SLX 1736-269 in 2003. Zero on the time axis corresponds to Aug. 23, 2003 - the begining of the Galactic Center ultra deep observations (proposal ID 0120213). Upper panel: thick horizontal line shows time intervals when the source SLX 1735-269 was in FOV of the JEM-X telescope. Values above horizontal dashed lines with arrows show effective exposure times during different sets of observations. Bottom panel: light curve of the source extracted from data of IBIS/ISGRI in the 18-60 keV energy band. Each point represents measurement averaged over $\sim 240 \mathrm{ks}$ (one revolution). Vertical solid lines on both panels indicate moments when type I bursts were detected. The length of this lines is proportional to the maximum value of the JEM-X count rate measured during the burst. The vertical arrow denotes the moment of the long burst beginning.

when the source was in the JEM-X fully coded field of view (FCFOV). Applying this simplest procedure to the Crab observations we found that the background-subtracted count rate of Crab is stable within $\sim 20 \%$. Therefore using values of count rates of the Crab nebula and pulsar in different energy bands and the background count rates in the same energy bands we can get the absolute flux of a source in the FCFOV of JEM-X with a reasonable accuracy.

For the spectral analysis we used a data product of OSA-4.0 (level SPE). The extensive study of the Crab nebula observations showed that the software allows us to reconstruct the shape of the source spectrum rather well while absolute fluxes are not reliable. Thus in the subsequent spectral analysis we used spectra produced by OSA-4.0, but renormalize them to the fluxes obtained from lightcurves (see above). The spectral analysis of the data obtained in the "restricted imaging mode" is not possible with OSA-4.0, therefore in this case we constructed source spectra from lightcurves in several broad energy bands.

The IBIS/ISGRI data analysis was done with the software developed by Eugene Churazov at the Space Research Institute, Moscow (Revnivtsev et al. 2004). This software provides the 


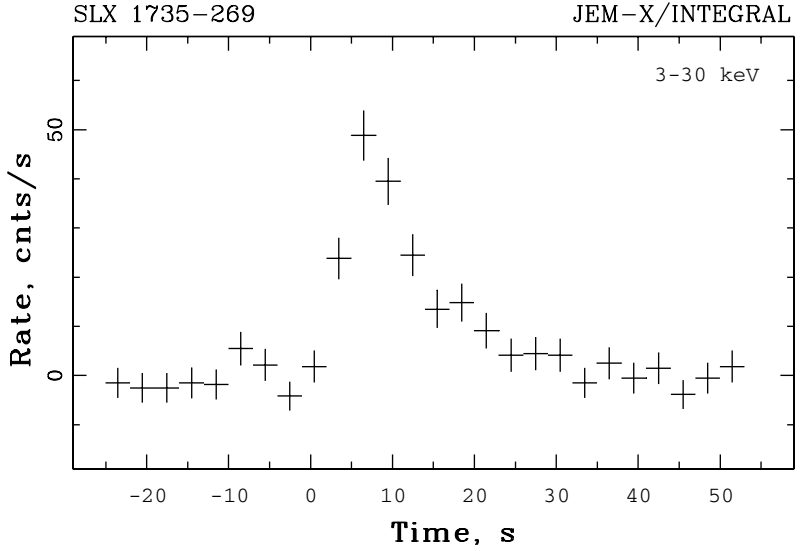

Fig. 2. Profile of the typical X-ray burst observed by INTEGRAL/JEM-X from SLX 1735-269 on Sept. 20, 2003 22:01:37 (UT).

Table 1. Bursts detected with INTEGRAL/JEM-X from the neutron star binary SLX 1735-269 during Galactic Center observations in 2003.

\begin{tabular}{ccccc}
\hline \hline$\#$ & $\begin{array}{c}\text { Start time } \\
\text { UT }\end{array}$ & $\begin{array}{c}\text { Peak flux }^{a}, \\
\text { Crab units }\end{array}$ & $\begin{array}{c}\tau^{a} \\
\text { s }\end{array}$ & $\begin{array}{c}\text { Burst } \\
\text { type }\end{array}$ \\
\hline 1 & Apr. 15, 09:48:35 & $0.87 \pm 0.17$ & $8.3 \pm 0.9$ & ordinary \\
2 & Sep. 15, 17:35:14 & $2.1 \pm 0.4$ & $>900$ & long \\
3 & Sep. 18, 21:50:53 & $0.97 \pm 0.19$ & $6.5 \pm 0.6$ & ordinary \\
4 & Sep. 20, 22:01:37 & $0.83 \pm 0.16$ & $7.4 \pm 0.5$ & ordinary \\
5 & Sep. 21, 10:37:15 & $0.63 \pm 0.12$ & $5.2 \pm 1.0$ & ordinary \\
6 & Sep. 23, 23:13:05 & $0.68 \pm 0.13$ & $9.5 \pm 1.3$ & ordinary \\
\hline
\end{tabular}

${ }^{a}$ In energy range $3-20 \mathrm{keV}$.

absolute values of source fluxes in a wide energy band with $10 \%$ systematic uncertainty.

Data of Rossi X-ray Timing Explorer (RXTE, Bradt et al. 1993) observations that we used for the construction of SLX 1735-269 broadband spectra were reduced with the help of standard tasks of the LHEASOFT 5.3 package.

Throughout the paper we use $1 \sigma$ confidence level of uncertainties.

\section{Results}

During all observation's 6 type I X-ray bursts were detected from SLX 1735-269 (see Fig. 1 and Table 1). All of them with one exception have properties similar to that observed by BeppoSAX/WFC (Bazzano et al. 1997; Cocchi et al. 1998). A typical profile of one of them (burst number \#4 from the Table 1) is presented in Fig. 2. All bursts demonstrated an exponential decay in the energy range $3-20 \mathrm{keV}$ with comparable e-folding times. These e-folding times along with $1 \sigma$ uncertainties are given in the Table 1. In this table we also present the peak fluxes of all bursts in the energy band 3-20 keV expressed in Crab units. Typical energies released in these bursts were approximately $E_{\text {burst }} \sim$ few $\times 10^{39} \mathrm{erg}$ (here and later we assume a canonical value of $8.5 \mathrm{kpc}$ ). Unfortunately a detailed spectral analysis is not possible for these bursts due to limited statistics.

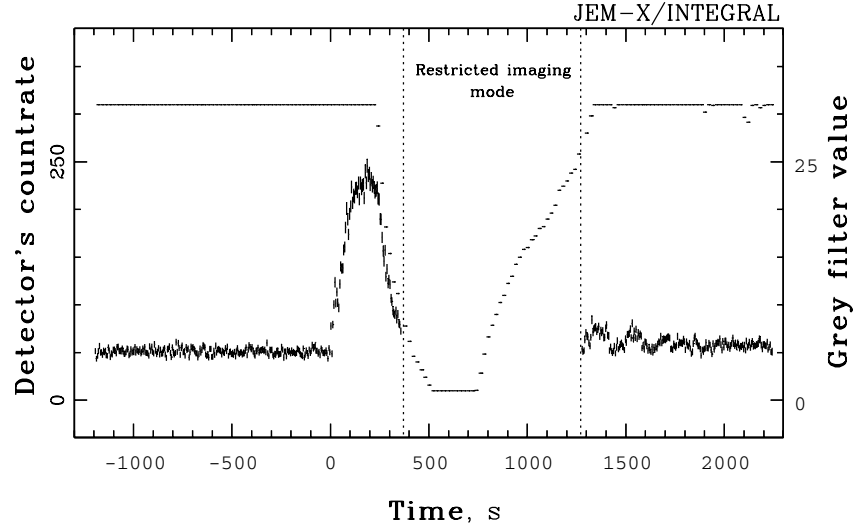

Fig. 3. Count rate collected from the whole JEM-X detector during the long burst. Solid step line denotes grey filter value changes. The zero time corresponds to the start of the burst.

An unusually long burst was observed on Sept. 15, 2003 at 17:35:14 UT during ultra deep Galactic Center observations. The count rate measured from the whole JEM-X detector and the grey filter factor during this observation are presented in Fig. 3.

\subsection{Profile of the long burst}

In Fig. 4 we present the profile of the long burst in different energy bands obtained from JEM-X data after the correction for the grey filter. Light curves in the subbands are background subtracted. The moment " 0 " corresponds to Sept. 15, 2003 17:35:14.0 (UT). The burst has a total duration of more than $2 \mathrm{ks}$ and its profile demonstrates several notable features. To emphasize these features we split the burst into four time intervals and plot each interval with its own time scale. The figure shows that the burst started with a short burst-like event ("precursor") that has a duration about $\sim 2 \mathrm{~s}$ and which was more powerful in the soft energy bands. For $\sim 8 \mathrm{~s}$ after the precursor the source flux was below the detectable level. Such "gaps" are typical for bursts with an extreme photospheric radius expansion and can be interpreted in terms of the cooling of the neutron star photosphere during its expansion. After this "gap" the flux of the source rises again to the maximum during $\sim 100-450$ s depending on the energy band. Such a behavior reflects a strong change of the source hardness and is also typical for bursts with photospheric radius expansion (a contraction phase). The source intensity decay after $\sim 450 \mathrm{~s}$ can be characterized by an e-folding time of $\tau=250 \pm 20 \mathrm{~s}$ in the energy band $2-7 \mathrm{keV}$ and $\tau=150 \pm 15 \mathrm{~s}$ in the $15-23 \mathrm{keV}$ energy band.

In the tail of the burst flux profile quasi-periodic oscillations can be seen on the time scale of $\sim 100 \mathrm{~s}$. Such oscillations are practically not visible in the harder energy band (10-23 keV) which indicates that the amplitude of these variations decreases with the energy. The time scale and energy dependence of these oscillation are very similar to those of $\mathrm{mHz}$ QPOs, seen in some other X-ray bursters (Revnivtsev et al. 2001). In that paper it is proposed that such oscillations can be caused by some special regime of nuclear burning on the neutron star surface. 


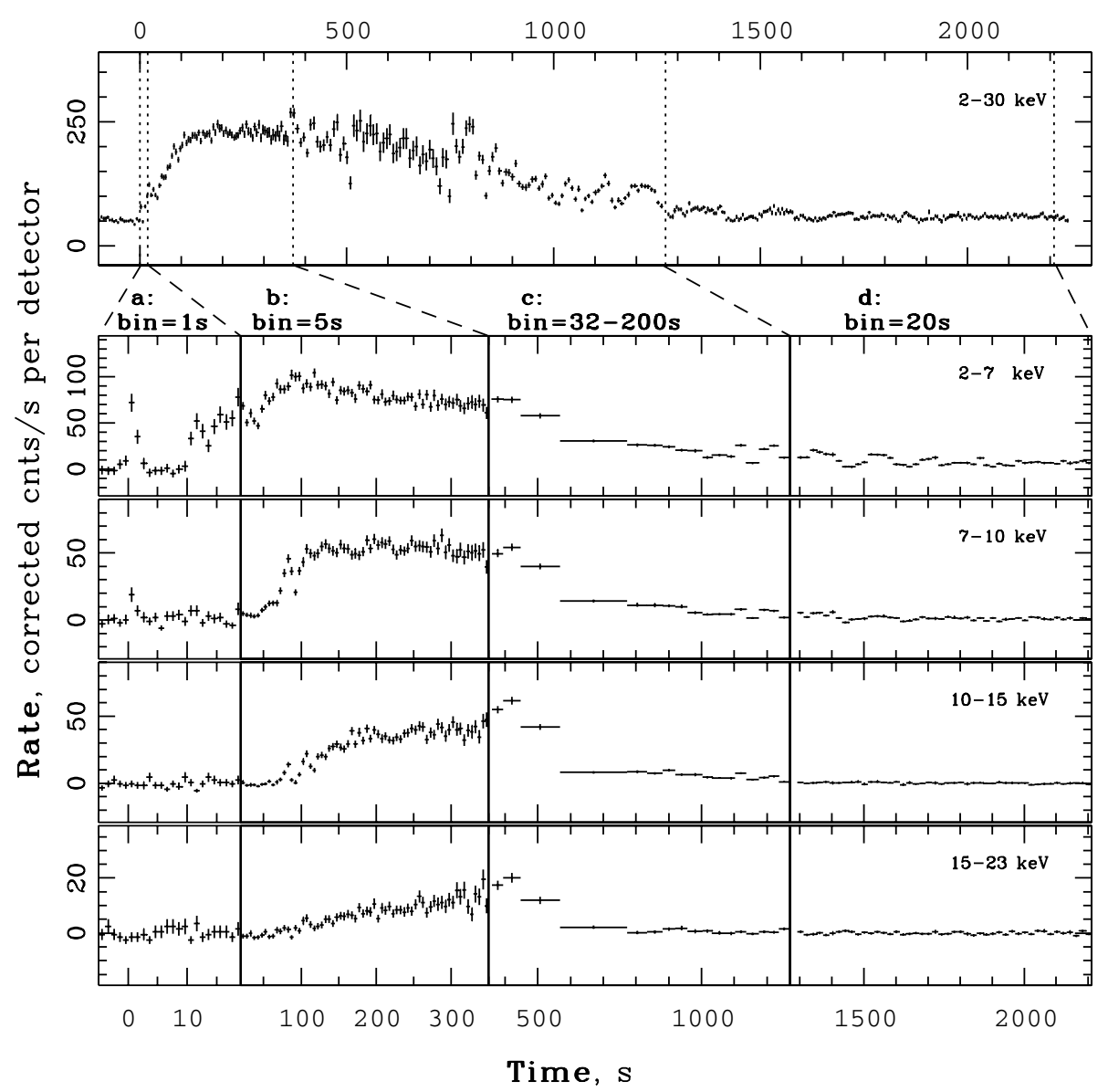

Fig. 4. Temporal profiles of the long burst measured by JEM-X in different energy bands. All light curves are corrected by the grey filter factor. Time binning for the light curve in the broad energy band (upper panel) is $5 \mathrm{~s}$ for the "full imaging mode" and $8 \mathrm{~s}$ for the "restricted mode". Time binning for other curves is indicated in the figure.

\subsection{Spectral evolution during the burst}

For the spectral analysis we extracted a source spectrum during a $2 \mathrm{~s}$ time interval covering the precursor, 17 consecutive spectra started from $\sim 20$ th $\mathrm{s}$ with an integration time of $20 \mathrm{~s}$, and 6 spectra in the restricted imaging mode. All obtained spectra were fitted by a blackbody model in the 3-20 keV energy band.

In the two upper panels of Fig. 5, the evolution of burst fluxes in the JEM-X 7-10 keV and ISGRI/IBIS $18-22 \mathrm{keV}$ energy bands are shown in mCrab units. In the middle panel we present the bolometric luminosity of the source (in units of $10^{38} \mathrm{erg} \mathrm{s}^{-1}$ ) calculated from the blackbody spectral model as a function of time. On this panel we can see that the source luminosity reached the Eddington limit at $\sim 100$ th $\mathrm{s}$ after the beginning of the burst. Such a long rise time is larger than that of typical powerful bursts with photospheric radius expansion (see e.g. Kuulkers et al. 2003, and references therein). It is unlikely that such behavior is caused by unusual chemical composition of the burning fuel or slow propogation of a thermonuclear burning front along the neutron star surface because the burst started with the very sharp rise (see the burst profile in 2-7 keV energy band, Fig. 4). After this rise the temperature of the X-ray emission strongly dropped (down to $\sim 1 \mathrm{keV}$ ) because of photospheric radius expansion and it is likely that we could miss some part of the source flux using our hard energy band ( $>2-3 \mathrm{keV})$. On the other hand it cannot be excluded that during the long burst we have a set of additional (triggered) thermonuclear bursts on the surface of the neutron star, which led to the long rise time to the maximum of our estimated bolometric flux. Indirect support for such a hypothesis can be found from the fact that we seen the radius expansion on the $\sim 40$ th $\mathrm{s}$ after the begining of the long burst.

The fit of the spectrum obtained during the precursor by this model gives the following parameters: $T_{b b}=1.1 \pm 0.2 \mathrm{keV}$ and $R_{b b}=26 \pm 7 \mathrm{~km}$. Such large uncertainties are caused by the limited sensitivity of JEM-X for such weak and soft events. During the next $\sim 8 \mathrm{~s}$ after the precursor the flux from the source was not detected. The evolution of the model parameters during the following $\sim 1000 \mathrm{~s}$ is presented in two lower panels of Fig. 5.

\section{Discussion}

\subsection{Bursts and persistent flux}

Before INTEGRAL only one type I X-ray burst had been detected from SLX 1735-269. This burst had a duration of $\sim 30$ s (e-folding decay time $\sim 10 \mathrm{~s}$ ) and a peak flux of approximately 900 mCrab (Bazzano et al. 1997; Cocchi et al. 1998) which translates into the energy emitted in the burst of a 


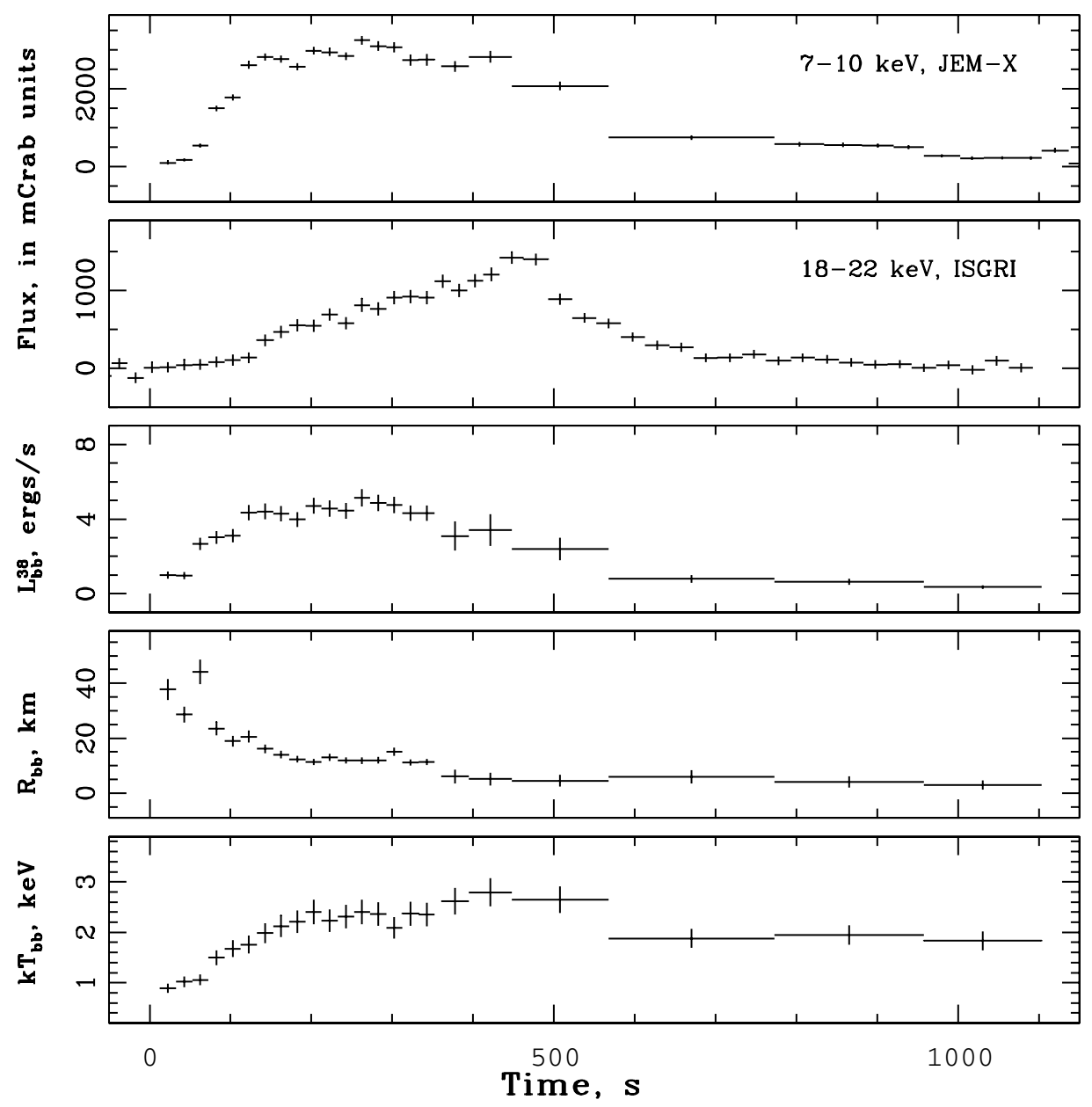

Fig. 5. Evolution of SLX 1735-269 X-ray fluxes, measured by JEM-X and IBIS telescopes in 7-10 and 18-22 keV energy bands, respectively; its bolometric luminosity, the radius of photosphere and its effective temperature during the long burst (from the black body fit to the spectra).

few $\times 10^{39} \mathrm{erg}$ (assuming a source distance of $8.5 \mathrm{kpc}$ ). Such an energy release and decay time are typical of the helium burning regime (see e.g. Fujimoto et al. 1981; Bildsten 1998).

INTEGRAL observations of the source in 2003 contain approximately 1.8 million seconds of exposure time for the JEM$\mathrm{X}$ monitor. During this exposure we detected in total $6 \mathrm{X}$-ray bursts. One of them was extremely powerful and long, while the others were similar to the one observed by BeppoSAX. In Fig. 1 we can distinguish two different intervals: from day 0 to 23 , when no bursts were detected and from day 23 to 33 when 4 short bursts and one long and poweful burst were detected. Using the JEM-X data and taking into account the data gaps we can estimate the $90 \%$ confidence upper limit on the short burst rate as 0.2 bursts per day for the first interval and the rate of short bursts as $0.5 \pm 0.1$ bursts per day for the second one. We can also estimate the upper limit of the rate of long burst generation (like the detected one). But in this case we can use not only JEM-X data but also the data from IBIS/ISGRI (which have a larger field of view and therefore a larger effective exposure) because such bursts are bright enough to be detected also in hard X-rays (18-30 keV). Taking all these into account our estimations of the long bursts rate are: 0.1 long bursts per day for the first interval.
Several short consecutive bursts allow us to estimate the burning regime parameter $\alpha$, the ratio between accretion energy released between bursts to the nuclear energy released in the burst. This parameter reflects the composition of the burning fuel (see e.g. the review Lewin et al. 1993). Using the set of almost consecutive bursts detected from day 25 to day 33 (in the units of Fig. 1) we estimated $\alpha=85 \pm 30$. Such values of $\alpha$ and bursts profiles and longevities are typical of the almost pure helium burning although hydrogen burning is not excluded (Fujimoto et al. 1981; Lewin et al. 1993; Bildsten 1998; Woosley et al. 2004).

If we assume that the luminosity of the source reaches the Eddington limit during the long burst we can estimate the distance to the source as from $6.0 \pm 1.2 \mathrm{kpc}$ for the pure helium atmosphere and up to $13 \pm 3 \mathrm{kpc}$ for the pure hydrogen atmosphere. In previouse works (see e.g. Cocchi et al. 1998) this distance was estimated as $8.5 \mathrm{kpc}$ which is in agreement with our range of distances.

In order to obtain the best estimate of the source luminosity and accretion rate we should consider the broadband spectrum because the hard X-ray information alone is not a good estimator of the bolometric luminosity of neutron stars (e.g. Barret 2001). To reconstruct the source broadband spectrum we included data of the RXTE observatory in our analysis. 


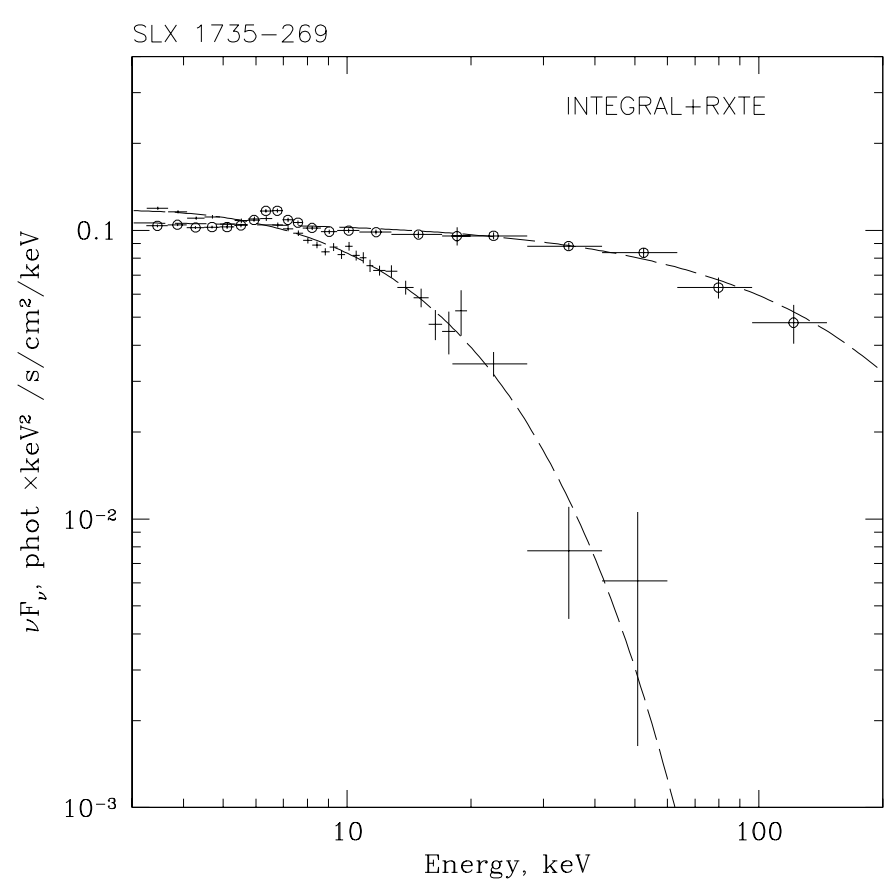

Fig. 6. Spectra of persistent X-ray emission of SLX 1735-269 at different states. Crosses denote the spectrum in the soft state (low hard X-ray flux), open circles denote the X-ray spectrum of SLX 1735-269 with high hard X-ray flux. All spectral points above $20 \mathrm{keV}$ are based on INTEGRAL/IBIS data, spectral points at energies lower $20 \mathrm{keV}$ taken from RXTE/PCA data. Dashed lines show spectra best fit models in the form of a power law with an exponential cutoff at high energies.

One observation of SLX 1735-269 was performed as part of a coordinated INTEGRAL+RXTE observational campaign of the system. At that time the source spectrum was hard, with a photon index of $\Gamma=2.0 \pm 0.1$ and an exponential cutoff at energy $E_{\text {cut }}=150_{-10}^{+50} \mathrm{keV}$ (see Fig. 6). The model broadband (0.5-100 keV) unabsorbed flux of the source was $\sim 5.4 \times 10^{-10} \mathrm{erg} \mathrm{s}^{-1} \mathrm{~cm}^{-2}$ that corresponds to a source luminosity of $L_{\mathrm{x}} \sim 4 \times 10^{36} \mathrm{erg} \mathrm{s}^{-1}$. During the period of low hard X-ray flux the source has a completely different spectrum. INTEGRAL observations show that the spectrum became much softer than it was during the period of the high hard X-ray flux. We searched the public RXTE data archive in order to find the source spectrum similar to that observed by INTEGRAL during the period of low hard X-ray (18-60 keV) flux. RXTE observations of SLX 1735-269 performed on Feb. 14, 2003 perfectly match this criterion. The total broadband $(0.5-100 \mathrm{keV})$ flux of the source in this state increased and the cutoff energy strongly diminished $\left(E_{\text {cut }}=11 \pm 1 \mathrm{keV}\right)$ in a comparison to the period of high hard X-ray flux (Fig. 6). The broadband (0.5$100 \mathrm{keV}$ ) flux in this state was $\sim 6.2 \times 10^{-10} \mathrm{erg} \mathrm{s}^{-1} \mathrm{~cm}^{-2}$ corresponding to a luminosity $L_{\mathrm{x}} \sim 5 \times 10^{36} \mathrm{erg} \mathrm{s}^{-1}$.

Note that the observed source luminosities differ only slightly while its spectral shape and burst properties differ dramatically. During the soft state, type I bursts occur quite often - the recurrence time between the bursts is approximately 0.5-2 days. In contrast, the recurrence time strongly increases, $\Delta t_{\mathrm{b}}>11$ days, when the spectrum is hard. This can indicate that the source persistent accretion rate is on the border between two distinct modes of nuclear burning. Theory stats that such a transition should occur approximately at the value of $\sim 10^{3} \mathrm{~g} \mathrm{~s}^{-1} \mathrm{~cm}^{-2}$ (e.g. Bildsten 1998). The persistent mass accretion rate on the neutron star in SLX 1735-269 (assuming an $\eta \sim 20 \%$ mass to the energy conversion coefficient and $L=\eta \dot{M} c^{2}$ ) is $\dot{M} \sim 2 \times 10^{16} \mathrm{~g} \mathrm{~s}^{-1}$. The corresponding accretion rate per unit area is $\dot{M} \sim 1.5 \times 10^{3} \mathrm{~g} \mathrm{~s}^{-1} \mathrm{~cm}^{-2}$ (assuming a $10 \mathrm{~km}$ radius of the neutron star). Therefore, we have a system in which the accretion rate is on the border of two regimes of nuclear burning. Note that this supports the assumption of the source distance of $8.5 \mathrm{kpc}$.

Short bursts with an e-folding time of $\tau \sim 10 \mathrm{~s}$ are rather typical among type I bursters. Very long bursts, with a duration more than $1000 \mathrm{~s}$ are rare and there are currently two possible scenarios for such bursts - the burning of a large pile of hydrogen and helium fuel, or the carbon flash (see e.g. Kuulkers et al. 2002a).

\subsection{Carbon flash scenario}

The source accretion rate per unit area $\dot{M} \sim 1.5 \times 10^{3} \mathrm{~g} \mathrm{~s}^{-1} \mathrm{~cm}^{-2}$ does not allow the burning of $\mathrm{He}$ in a stable manner (Bildsten 1998). Therefore the accumulation of a large amount of carbon cannot be done via this process and most of the carbon fuel needed to produce the burst should be accreted from a companion star. An estimation of the energy released in the burst gives $E_{\text {burst }}=2 \pm 0.4 \times 10^{41} \mathrm{erg}$. To provide this energy through carbon burning, $M \sim 5 \times 10^{23} \mathrm{~g}$ of carbon is needed (for a nuclear energy release of $\simeq 5.6 \times 10^{17} \mathrm{erg} \mathrm{g}^{-1}$, Cumming \& Bildsten 2001). To accumulate such an amount of carbon via the accretion from the main sequence secondary star we need more than $10^{9} \mathrm{~s}$ (assuming a solar abundance of ${ }^{12} \mathrm{C}$ in the accreted matter). Therefore the probability of detecting one burst in our set of observations (an effective exposure time $\sim 2 \times 10^{6} \mathrm{~s}$ ) is less than one tenth of percent. An additional argument against the hypothesis that this burst is a "superburst" is the low source average accretion rate, less than $10 \%$ of the Eddington limit (see Cumming \& Bildsten 2001). Thus we conclude that the long burst from SLX 1735-269 is unlikely to result from a carbon shell flash.

\section{3. $\mathrm{He} / \mathrm{H}$ flash scenario}

For such low accretion rates X-ray bursts can be caused by a mixed hydrogen and helium burning triggered by hydrogen ignition accelerated by electron capture processes (see e.g. Fushiki et al. 1992; Bildsten 1998; Bildsten \& Cumming 1998; Kuulkers et al. 2002a). The nuclear energy release per gram of such fuel is a few times $10^{18} \mathrm{erg} \mathrm{g}^{-1}$ (depending on the fuel composition). A mass of $\sim 10^{23}$ g needs to be accreted to provide the fuel which will take $\sim 3 \times 10^{6} \mathrm{~s}$ at the above-mentioned accretion rate. This recurrence time is comparable with the exposure of our observations. The critical surface density necessary to make electron capture processes very effective is of the order of $y \sim 10^{10} \mathrm{~g} \mathrm{~cm}^{-2}$, which means that the accreted mass $M \sim 10^{23} \mathrm{~g}$ of the matter should be involved. This value again 
is compatible with the observed value of the mass burned in the long burst.

The length of the burst in this scenario mainly depends on the limiting (Eddington) luminosity. It is governed by the energy budget of the burst divided by the Eddington luminosity on the neutron star. The stability of the source luminosity over a few hundreds of seconds at the peak of the burst (Fig. 5) supports this assumption.

\section{Conclusions}

Thus, we conclude that the long burst detected from SLX 1735269 most likely is a result of the unstable burning of a large amount of mixed hydrogen and helium accelerated by electron capture processes. A similar long burst was observed by BeppoSAX from other faint neutron star binaries (Kuulkers et al. 2002b; in 't Zand et al. 2002).

The detected long burst occurred shortly after the source transition from the hard/low state to the soft/high state. It is likely that this can be the result of the rapid accumulation of a critical amount of matter on the neutron star surface for the start of the bursting activity. It is very unlikely that the burst provoked the state transition because the transition occurred before the burst.

Finally we would like to emphasize two features which make the long burst of SLX 1735-269 unusual even within the limited number of long bursts of other X-ray bursters. The first one is the rather slow rise to the peak after the precursor. One of the possible reasons for this effect could be the existence of an additional burst on the surface of the neutron star between the precursor and the moment when the photosphere of the neutron star collapsed after the primary burst. Indirect support for such an hypothesis can be found from the fact that the photospheric radius expansion occurred at the $\sim 40$ th $\mathrm{s}$ after the begining of the long burst. Another possible explanation for this effect could be the deviation of the spectra's shapes from a powerlaw model and in this case we underestimate the bolometric luminosity of the source. The second effect is the X-ray flux variations on the time scale of $\sim 100 \mathrm{~s}$ in the tail of the burst. The amplitude of these variations decreases toward high energies and these oscillations are not visible in the harder energy band (10-23 keV). The time scale and energy dependence of the oscillations are very similar to those of $\mathrm{mHz}$ QPOs, seen in some other X-ray bursters (Revnivtsev et al. 2001). In that paper the authors proposed that such oscillations can be caused by a special regime of nuclear burning on the neutron star surface.

Acknowledgements. The authors thank E. Churazov for the developing of the methods of analysis of the IBIS data and software. We would like to thank Prof. L. Bildsten for very useful discussions and comments. This research has made use of data obtained through the INTEGRAL Science Data Center (ISDC), Versoix, Switzerland, Russian INTEGRAL Science Data Center (RSDC), Moscow, Russia, and High Energy Astrophysics Science Archive Research Center Online Service, provided by the NASA/Goddard Space Flight Center. This work was supported by RFBR grant 04-02-17276, grants of Minpromnauka NSH-2083.2003.2 and 40.022.1.1.1103.

\section{References}

Barret, D. 2001, Adv. Sp. Res., 28, 307

Bazzano, A., Cocchi, M., Ubertini, P., et al. 1997, IAU Circ., 6668

Bildsten, L. 1998, in The many faces of neutron stars, ed. J. Bucheri, J. van Paradijs, \& M. A. Alpar (Dordrecht: Kluwer), 419

Bildsten, L., \& Cumming, A. 1998, ApJ, 506, 842

Bradt, H., Rotshild, R., \& Swank, J. 1993, A\&AS, 97, 355

Chernyakova, M., \& Kretschmar, P. 2004, JEM-X analysis user manual

Cocchi, M., in 't Zand, J., Bazzano, A., et al. 1998, Results from BeppoSAX and RXTE: The active X-ray Sky, Nucl. Phys. B, Proc. Suppl., 69/1-3, 232

Cornelisse, R., Heise, J., Kuulkers, E., et al. 2000, A\&A, 357, L21

Cumming, A., \& Bildsten, L. 2001, ApJ, 559, L127

David, P., Goldwurm, A., Mukarakami, T., et al. 1997, A\&A, 322, 229

Fujimoto, M., Hanawa, T., \& Miyaji, S. 1981, ApJ, 246, 267

Fushiki, I., Taam, R., Woosley, S., \& Lamb, D. 1992, ApJ, 390, 634

Goldwurm, A., Vargas, M., Paul, J., et al. 1996, A\&A, 310, 857

Grebenev, S., Pavlinsky, M., \& Sunyaev, R. 1996, The Transparent Universe, Proc. of 2nd INTEGRAL Workshop, ESA SP-382, 183

Hansen, C. J., \& van Horn, H. M. 1975, ApJ, 195, 735

in 't Zand, J. 1992, Ph.D. Thesis, Utrecht University

in 't Zand, J., Verbunt, F., Kuulkers, E., et al. 2002, A\&A, 389, L43

in 't Zand, J. J. M., Cornelisse, R., \& Cumming, A. 2004

[arXiv: astro-ph/0407083]

Kuulkers, E., in 't Zand, J. J. M., van Kerkwijk, M. H., et al. 2002a, A\&A, 382, 503

Kuulkers, E., Homan, J., van der Klis, M., et al. 2002b, A\&A, 382, 947

Kuulkers, E., den Hartog, P. R., in 't Zand, J. J. M., et al. 2003, A\&A, 399, 663

Lewin, W., van Paradijs, J., \& Taam, R. 1993, SSRv., 223

Lund, N., Brandt, S., Budtz-Joergesen, C., et al. 2003, A\&A, 411, L231

Pavlinsky, M. N., Grebenev, S. A., \& Sunyaev, R. A. 1992, Astr. Lett., 18, 116

Pavlinsky, M. N., Grebenev, S. A., \& Sunyaev, R. A. 1994, ApJ, 425, 110

Revnivtsev, M., Churazov, E., Gilfanov, M., \& Sunyaev, R. 2001, A\&A, 372, 138

Revnivtsev, M., Sunyaev, R., Varshalovich, D., et al. 2004, Astr. Lett., 30, 382

Skinner, G. K., Willmore, A. P., Eyles, C. J., et al. 1987, Nature, 330, 544

Strohmayer, Tod, E., \& Brown Edward, F. 2002, ApJ, 566, 1045

Ubertini, P., Lebrun, F., Di Cocco, G., et al. 2003, A\&A, 411, L131

Winkler, C., Courvoisier, T. J.-L., Di Cocco, G., et al. 2003a, A\&A, 411, L1

Winkler, C., Gehrels, N., Schönfelder, V., et al. 2003b, A\&A, 411, L349

Woosley, S., Heger, A., Cumming, A., et al. 2004, ApJS, 151, 75 